\title{
Crystal Habits and the Variation in Surface Energy Heterogeneity
}

\author{
Raimundo Ho, ${ }^{\dagger}$ David A. Wilson, ${ }^{\ddagger}$ and Jerry Y.Y. Heng ${ }^{\dagger, *}$ \\ ${ }^{\dagger}$ Surfaces and Particle Engineering Laboratory, Department of Chemical Engineering, Imperial \\ College London, South Kensington Campus, London SW7 2AZ, United Kingdom \\ ${ }^{\ddagger}$ AstraZeneca, Pharmaceutical and Analytical R\&D, Macclesfield, Cheshire SK10 2NA, United \\ Kingdom
}

\begin{abstract}
The anisotropicity of crystal facets means that the overall adhesion property or wetting behavior of a crystalline batch are dependent on the crystal habits of the constituent particles. A recently developed method based on Inverse Gas Chromatography at finite concentration was applied to study the variation in surface energy heterogeneity of the $\beta$ polymorph of D-mannitol exhibiting different crystal habits. The dispersive surface energy distributions were measured for D-mannitol crystals with various aspect ratios as confirmed by Dynamic Image Analysis. D-mannitol with dissimilar crystal aspect ratios have similar range of surface energies but are significantly different in the relative proportion of energy values. As the aspect ratio of D-mannitol decreased, there is a decrease in the overall contribution of the lower dispersive surface energy regions, which was attributed to the decrease in the proportion of the lowest energy crystal facet. This study confirms that crystal shape has a profound influence on the overall surface heterogeneity.
\end{abstract}

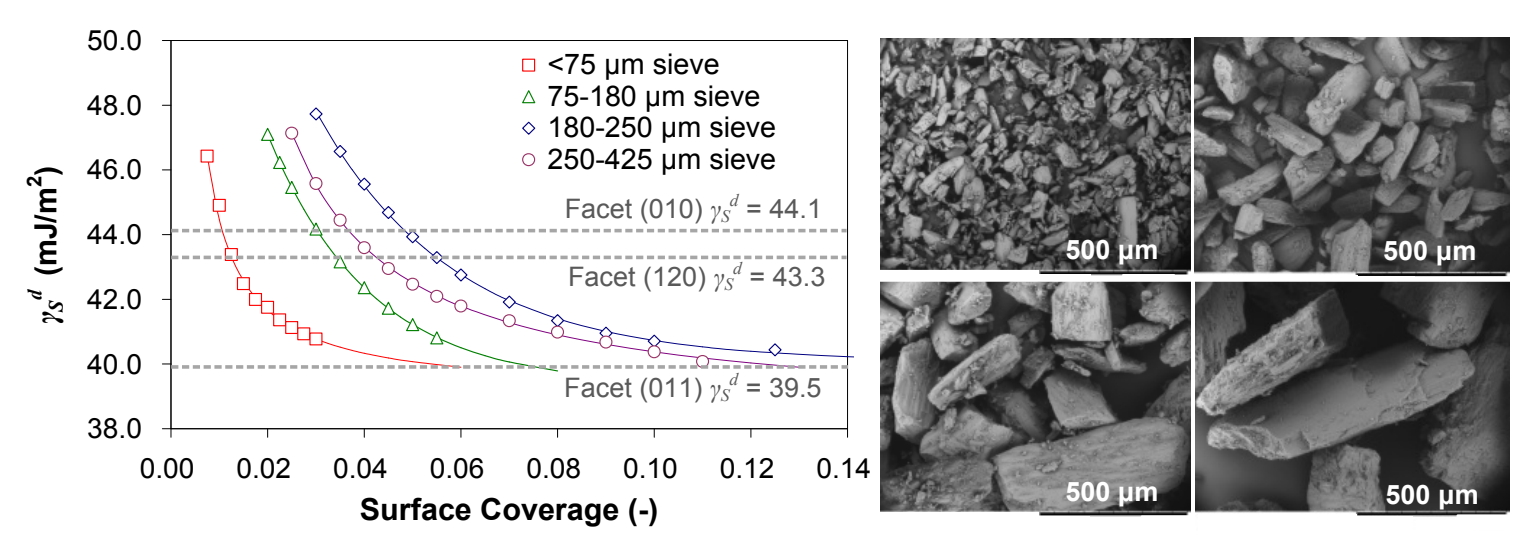

*To whom correspondence should be addressed:

Jerry Y.Y. Heng

Surfaces and Particle Engineering Laboratory, Department of Chemical Engineering, Imperial College London, South Kensington Campus, London SW7 2AZ, United Kingdom.

Tel: +44 (0)20 75940784 Fax: +44 (0)20 75945638 E-mail: jerry.heng@imperial.ac.uk 


\title{
Crystal Habits and the Variation in Surface Energy Heterogeneity
}

\author{
Raimundo Ho, ${ }^{\dagger}$ David A. Wilson, ${ }^{\dagger}$ and Jerry Y.Y. Heng ${ }^{\dagger, *}$ \\ ${ }^{\dagger}$ Surfaces and Particle Engineering Laboratory, Department of Chemical Engineering, Imperial \\ College London, South Kensington Campus, London SW7 2AZ, United Kingdom \\ ${ }^{\star}$ AstraZeneca, Pharmaceutical and Analytical R\&D, Macclesfield, Cheshire SK10 2NA, United \\ Kingdom \\ *To whom correspondence should be addressed: \\ Jerry Y.Y. Heng \\ Surfaces and Particle Engineering Laboratory \\ Department of Chemical Engineering \\ Imperial College London \\ South Kensington Campus \\ London SW7 2AZ \\ United Kingdom \\ Tel: +44 (0)20 75940784 \\ Fax: +44 (0)20 75945638 \\ E-mail: jerry.heng@imperial.ac.uk
}


ABSTRACT: The anisotropicity of crystal facets means that the overall adhesion property or wetting behavior of a crystalline batch are dependent on the crystal habits of the constituent particles. A recently developed method based on Inverse Gas Chromatography at finite concentration was applied to study the variation in surface energy heterogeneity of $\beta$ form of D-mannitol exhibiting different crystal morphologies. The dispersive surface energy distributions were measured for D-mannitol crystals exhibiting various aspect ratios as confirmed by Dynamic Image Analysis. D-mannitol with dissimilar crystal aspect ratios have similar range of surface energies but are significantly different in the relative proportion of energy values. As the aspect ratio of D-mannitol decreased, there was a decreasing shift in the overall contribution of the lower dispersive surface energy regions, which was attributed to the decrease in the proportion of the lowest energy crystal facet. This study confirms that crystal shape has a profound influence on the overall surface heterogeneity. 


\section{Introduction}

Crystals of the same polymorph may exhibit a variety of habits depending on the relative growth rates of individual facets, which are suggested to be dependent upon the intrinsic properties of the crystals, such as interplanar spacing and attachment energies, ${ }^{1,2}$ as well as external influences such as the solvent type ${ }^{3,4}$ and additives. ${ }^{5}$ The habit of crystals not only plays a key role in their powder behavior and physicochemical properties such as wettability, mechanical strength, dispersibility in suspensions, flowability and bulk density, ${ }^{6}$ but also their ease of processing and manufacturing, for instance, in drying, compaction ${ }^{7}$ and milling operations. ${ }^{8}$ In the case of solid-state pharmaceuticals, the crystal habits also determine the dissolution rates, ${ }^{9,10}$ drug delivery performance of the active ingredient in inhaled formulations, ${ }^{11}$ and the bioavailabilities of active ingredients. ${ }^{12}$ The engineering of materials to achieve a desired crystal size and shape distribution in order to provide end-use efficacy and down-stream processing efficiency has, therefore, been a subject of considerable interests for different chemical industries. ${ }^{13,14}$

It is known that the surface properties of organic crystalline materials are anisotropic: the surface properties or activities of organic crystal faces are directly related to the localized chemical functionality. The presence of a dominant crystal face may, therefore, determine the bulk physical and chemical properties of the material, which can also be affected by secondary processing operations, e.g. milling, resulting in the exposure of crystal facets or surface regions with dissimilar surface properties. ${ }^{15}$ For this reason, the crystal habit is an important determinant on the wetting 
behavior of a sample batch, because this behavior is influenced by the relative exposure of various indexed facets in the sample. More importantly, the overall surface chemistry of a crystalline solid is not just a reflection of the various crystal facets, but also the unique combination of impurities, growth steps, crystal edges, surface pores, local degree of crystallinity and surface functional groups on the surface. ${ }^{16}$ Changes in the material surface energetics were reported to profoundly influence adhesion, ${ }^{17}$ dissolution ${ }^{18}$ and granulation ${ }^{19}$ performances. Although the use of a single value average may provide first order estimation of the surface properties of the material, it may be inadequate to differentiate batch-to-batch variations which are, sometimes, undetectable by conventional techniques. The ability to quantify the compositions of different energy sites or facet-dependent surface energy can be extremely useful in the more accurate prediction of processing and formulation behavior, for instance, in the aerosolization performance in inhaler formulations, ${ }^{20}$ and the cohesion-adhesion balance between drug and excipient in mixing. ${ }^{17}$

In this work, a new approach involving inverse gas chromatography (IGC) to quantify small differences in surface energy due to crystal morphology was examined. A commonly used crystalline pharmaceutical excipient, D-mannitol $\left(\mathrm{C}_{6} \mathrm{H}_{14} \mathrm{O}_{6}\right)$, is the subject of the current investigation. D-mannitol is widely used in a variety of oral formulations because of its non-hygroscopicity, non-cariogenicity, sweetness, 'mouth feel' and cooling property in the oral cavity. $^{21,22}$ The thermodynamically stable $\beta$ polymorph crystallizes as a prismatic rod, and has been shown to exhibit anisotropic wettability in our earlier study. ${ }^{23}$ In the present work, the variation in $\gamma_{S}^{d}$ due to changes in the crystal habit of D-mannitol was investigated. 
Quantifying Surface Free Energy Distributions with IGC. The application of IGC to measure surface properties and surface energetics of pharmaceutical powders is well documented in the literature. ${ }^{24-26}$ Measurements of $\gamma_{S}^{d}$ in IGC experiments require the solid powder sample to be packed in a column where purely dispersive alkane vapour probes are injected via an inert carrier gas such as helium. By measuring the retention behavior of these probe molecules, the net retention volume $\left(V_{N}\right)$, which is a fundamental surface thermodynamic property, can be obtained and related to a range of surface properties such as surface energy heterogeneity and surface acid-base functionality.

The net retention volume is related to the experimental conditions and the retention time of the probe as shown in eq 1.

$$
V_{N}=\frac{j}{m} \cdot F \cdot\left(t_{R}-t_{0}\right) \cdot \frac{T}{273.15}
$$

where $m$ is the sample mass, $F$ is the carrier gas exit flow rate at standard temperature and pressure (STP), $T$ is the column temperature in $\operatorname{Kelvin}(\mathrm{K}), t_{R}$ is the retention time for interacting probe, $t_{0}$ is the mobile phase hold-up time and $j$ is the James-Martin correction, which corrects the retention time for the pressure drop along the column bed. The net retention volume of each alkane injection is related to the dispersive surface free energy component via

$$
R T \ln V_{N}=2 N_{A}\left(\gamma_{S}^{d}\right)^{1 / 2} a_{m}\left(\gamma_{L}^{d}\right)^{1 / 2}+K
$$

where $R$ is the universal gas constant, $K$ is a constant, $N_{A}$ is the Avogadro's number and $a_{m}$ is the cross sectional area of the adsorbate. A plot of $R T \ln V_{N}$ versus $a_{m}\left(\gamma_{L}^{d}\right)^{1 / 2}$ for the alkanes will be linear and $\gamma_{S}^{d}$ can then be calculated from the slope. ${ }^{27}$ 
The distribution of $\gamma_{S}^{d}$ of the test sample can be determined by injecting a series of concentration of alkanes into the sample column, i.e. an increase from low partial pressure up to saturation vapour pressure. In IGC finite concentration experiments, although the alkanes are injected at the same series of concentrations, their difference in molecular cross sectional area (due to increasing alkane chain length) and the difference in the equilibrium pressure inside the column (due to their different saturation vapour pressure) means that the retention volumes represent significantly dissimilar probe molecule surface coverage, therefore cannot be compared directly. However, if the retention volumes obtained from measurements at these different concentrations can be corrected for their corresponding surface coverages, then eq 2 can be applied to calculate the $\gamma_{S}^{d}$ value for each surface coverage, thereby resulting in a distribution of $\gamma_{S}^{d}$. A detailed description of the methodology and the treatment of the retention volumes to coincide their surface coverage is described in detail elsewhere. ${ }^{28,29}$ In the present study, this new methodology will be used to determine the surface energy distribution of various particulate D-mannitol samples exhibiting slight variation in their crystal habits.

Quantifiying Crystal Habit with Dynamic Image Analysis (DIA). Traditional methods of shape analysis would typically employ a form of static microscopy, being either optical or electron. With such techniques, due to the low number of particles imaged (typically $<100$ particles), data interpretations are highly subjective and prone to effects of specific orientation of the particles. Laser diffraction instruments have been used to obtain shape information, but these experiments are still premature for commercial implementation. ${ }^{30}$ With the advances in automated image analysis 
instrumentations, such as DIA in which the two-dimensional images of particles are captured in a fast moving air or liquid stream by a high-speed digital camera, both the particle size and shape can be measured with versatility and high reproducibility. In this study, the crystal habits of the particles are described in a reliable and statistically robust manner, due to the fact that DIA involves the measurement of several million crystals per batch in a random orientation. The direct relationship between crystal habit of D-mannitol as measured by the DIA technique and its surface energy heterogeneity is examined. 


\section{Experimental Section}

Materials. D-mannitol (Ph Eur Pearlitol ${ }^{\circledR} 160 \mathrm{C}$, Roquette, France) was used without further purification. The material was sieved using a base pan and 75, 180, 250 and $450 \mu \mathrm{m}$ stainless steel test sieves (Pascall Engineering, Suffolk, U.K.) to obtain four sieve fractions $(<75 \mu \mathrm{m}, 75-180 \mu \mathrm{m}$, $180-250 \mu \mathrm{m}$ and $250-450 \mu \mathrm{m})$ for $\gamma_{S}^{d}$, BET surface area, particle size and morphology analyzes.

Inverse Gas Chromatography (IGC). IGC experiments were conducted using an iGC 2000 (Surface Measurement Systems, London, U.K.) with a flame ionisation detector. Each sieve fraction of D-mannitol was packed into separate standard pre-silanized columns $(300 \times 4 \mathrm{~mm}$ ID) with silanzied glass wool at each end to prevent powder movement. Columns were filled with $2.2-2.4 \mathrm{~g}$ of material and then conditioned in-situ in the iGC with helium for $2 \mathrm{~h}$ at $303 \mathrm{~K}$ to remove any physisorbed water. Following pre-treatment, pulse injections using a $0.25 \mathrm{ml}$ gas loop at $303 \mathrm{~K}$ were performed. A series of purely dispersive n-alkane vapour probes (decane, nonane, octane, heptane) (HPLC grade, Sigma-Aldrich, Poole, U.K.) were injected at 0.03, 0.05, 0.10, 0.25, 0.50, $0.60,0.70,0.80$ and $0.95 p / p_{0}$ to determine the adsorption isotherms, and net retention volumes were determined using peak maximum analysis. Methane gas was injected at $0.10 \mathrm{p} / \mathrm{p}_{0}$ to determine column dead time. Helium, at a flow rate of $10 \mathrm{sccm}$, was used as the carrier gas for all injections. $V_{N}$ and adsorption isotherms were calculated using SMS-iGC Analysis Macros (version 1.2, Surface Measurement Systems, London, U.K.). 
Surface Area Analysis. The Brunauer-Emmett-Teller (BET) surface area was determined from nitrogen adsorption isotherms using a fully automated surface area analyzer Tristar 3000 (Micromeritics, Norcross, GA). Approximately 3-4 g of samples were pre-conditioned with helium purge at $60^{\circ} \mathrm{C}$ for at least $4 \mathrm{~h}$ prior to measurement.

Particle Size and Morphology Analysis. Particle size and aspect ratio of the samples were measured with a dynamic image analysis system QICPIC (Sympatec Ltd, Clausthal-Zellerfeld, Germany) fitted with a dry air disperser (RODOS, Sympatec) operating at a pressure of 0.5 bar and dry vibratory feeder system (VIBRI, Sympatec). The system operated with a pulsed light source with sub-nanosecond illumination, and the particles were imaged by a high speed camera with a frame rate of $400 \mathrm{fps}$ (frames per second). Images were analysed by the software WINDOX (version 5, Sympatec) to obtain maximal Feret diameter density distribution and aspect ratio of the feed particles. A minimum of $10^{6}$ particles per sample were characterized and the samples were run in triplicate.

Polymorph Identification. Powder X-ray diffraction analysis was carried out to identify the crystal structure of the powder sample using a X'Pert Pro diffractometer (PANalytical B.V., Almelo, The Netherlands) over the range of $10-50^{\circ} 2 \theta$ with a CuK $\alpha$ X-ray source at $40 \mathrm{kV}$ and $40 \mathrm{~mA}$.

SEM Images. SEM images were acquired with a tabletop microscope system TM-1000 (Hitachi, Tokyo, Japan) in the charge-up reduction mode. 


\section{Results and Discussion}

Crystal Habit. Kaminsky and Glazer ${ }^{31}$ reported that single crystals of $\beta$ form of D-mannitol crystallize from saturated water solution in the orthorhombic form with four $(z=4)$ molecules in each unit cell and of $\mathrm{P} 2{ }_{1} 2_{1} 2_{1}$ space group. The crystals consist of indexed facets $\{011\},\{010\}$, $\{120\},\{110\}$ and $\{210\}$ (Figure 1).
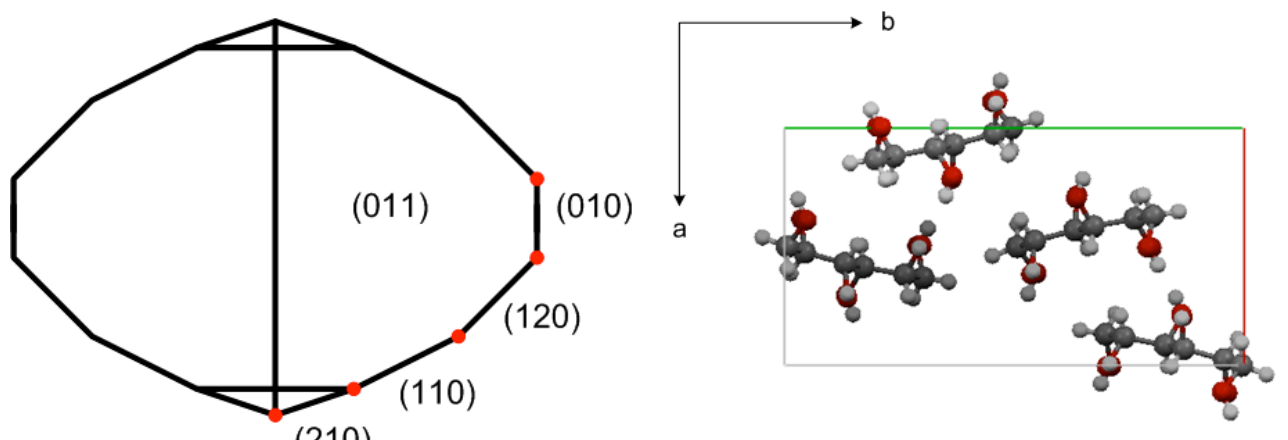

(210)

Figure 1. Crystal habit for $\beta$ form of D-mannitol with facets $\{011\},\{010\},\{120\},\{110\}$ and $\{210\}$ as reported by Kaminsky and Glazer. ${ }^{31}$

Using the same solvent, $\beta$ crystals of up to $60 \mathrm{~mm}$ length and a cross-section area of approximately $8 \mathrm{~mm} \times 8 \mathrm{~mm}$ were successfully crystallized from needle seeds by controlled cooling of the solvent in our earlier study. ${ }^{23}\{210\}$ and $\{110\}$ were found to diminish after long growth period, suggesting these facets are the fastest growing faces. Although the crystallization conditions for the commercially available powder sample used in this study are unknown, PXRD analysis ascertained that this sample is the $\beta$ polymorph (Figure 2), and also exhibits similar crystal 
habit as reported by Kaminsky and Glazer ${ }^{31}$ using SEM. The crystal habits of the four sieve fractions of D-mannitol, obtained by SEM, are displayed in Figure 3.

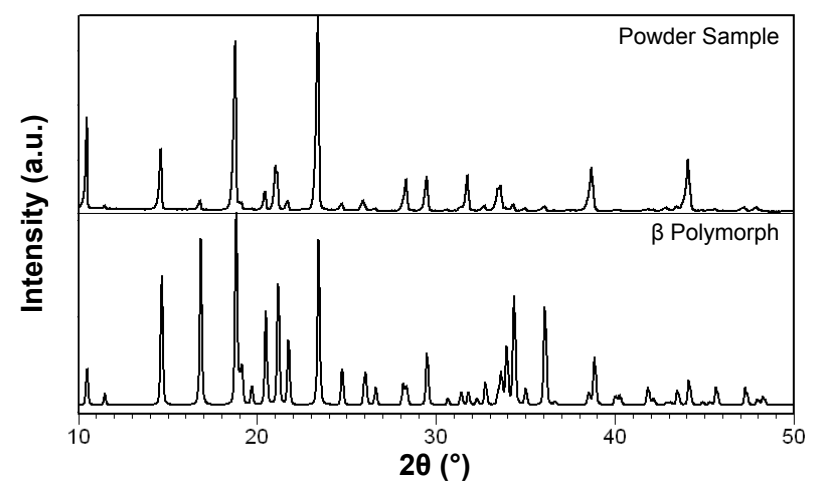

Figure 2. Comparison of PXRD patterns of the D-mannitol powder sample with the reported pattern by Walter-Levy. ${ }^{32}$
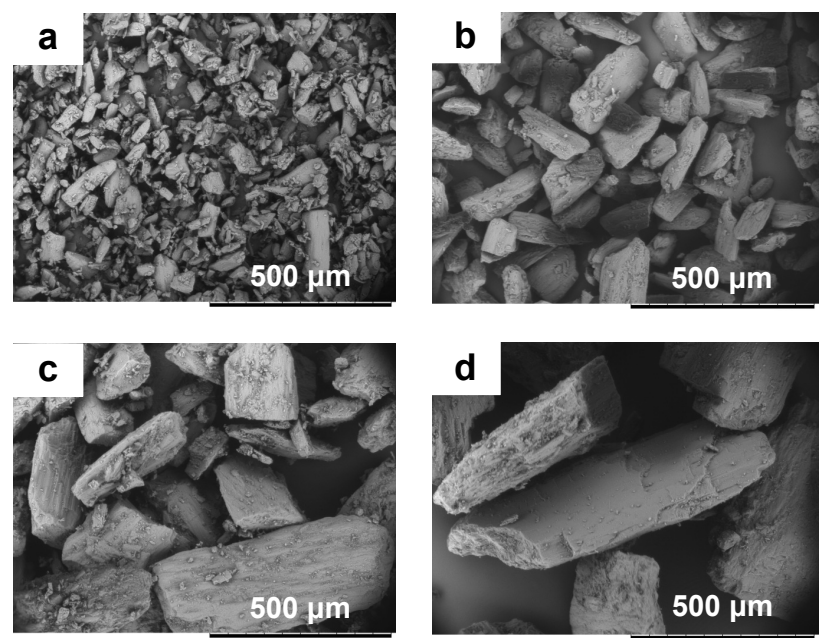

Figure 3. SEM images of Pearlitol ${ }^{\circledR} 160 \mathrm{C}$ sieved into 4 different fractions (a) $<75 \mu \mathrm{m}$ (b) $75-180$ $\mu \mathrm{m}$ (c) $180-250 \mu \mathrm{m}$ and (d) $250-425 \mu \mathrm{m}$.

The surface-area-weighted size distributions (Q2) of the four sieve fractions were determined from dynamic image analysis and are displayed in Figure 4. It can be seen that the peak of the 
distributions shifts to higher particle size as the sieve size increases, which is consistent with intuitive expectation. However, it should be noted that sieving would classify particles based upon the smallest projected diameter, i.e. the diameter of the sieve or the minimal Feret diameter. The maximal Feret diameter may, therefore, be a better representation of the particle size in a particular sieve fraction. As such, when converting the projected diameter into the maximal Feret diameter in DIA, it is expected that some particles larger than the stipulated sieve size will be obtained, as is the case for all 4 fractions in Figure 4. Further analysis of the Q2 size data also indicated that the sieving was not efficient enough to remove all particles smaller than the lower sieve size for the sieve cuts.
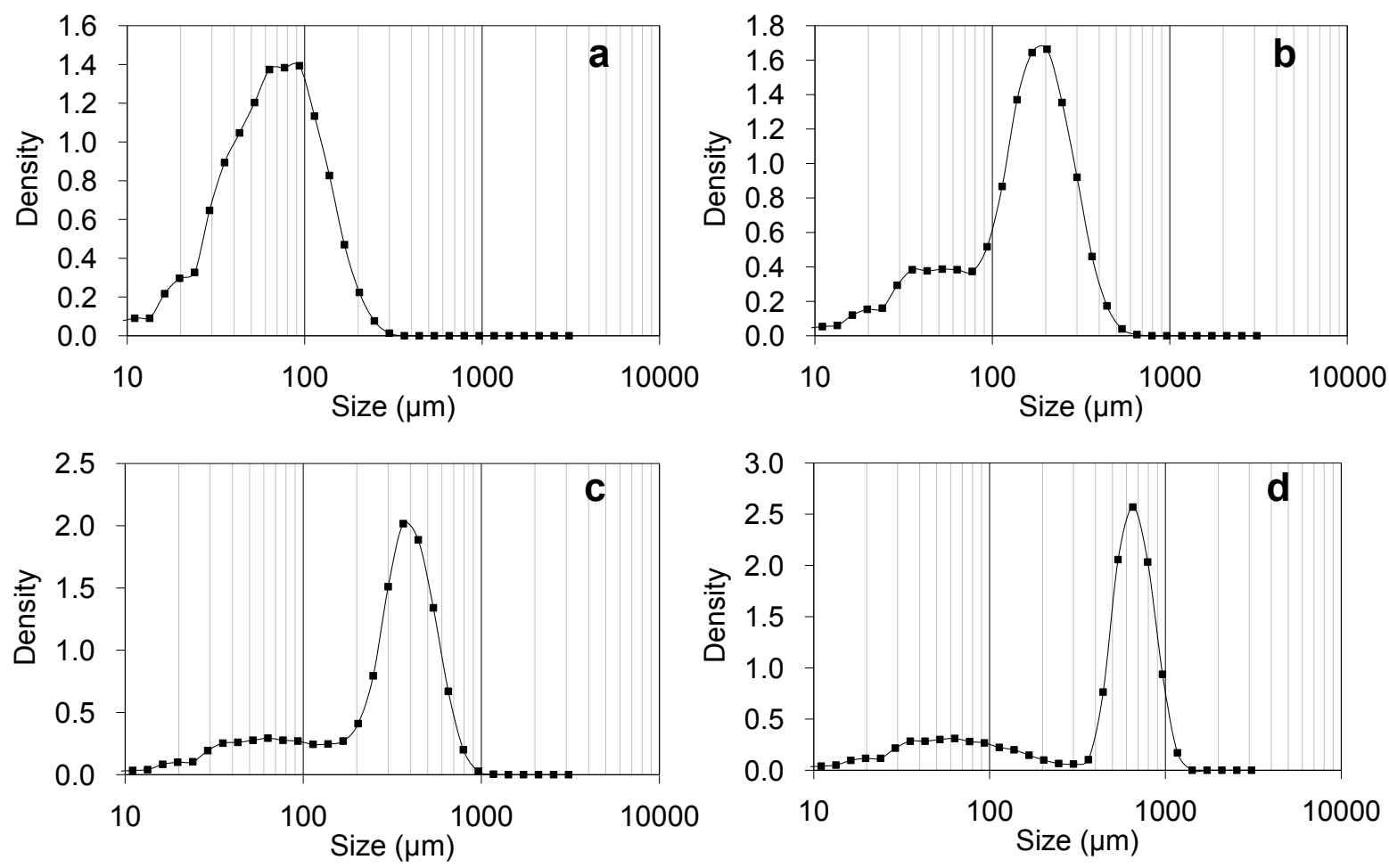

Figure 4. Particle size distributions of D-mannitol weighted by surface area for (a) $<75 \mu \mathrm{m}$ micron sieve fraction (b) $75-180 \mu \mathrm{m}$ sieve fraction (c) $180-250 \mu \mathrm{m}$ sieve fraction and (d) $250-425 \mu \mathrm{m}$ 
sieve fraction.

The aspect ratio, which is defined as the ratio of the minimal to the maximal Ferret diameter of the particle, is a commonly-used shape parameter for needle-shaped crystals such as D-mannitol. In order to provide a representative aspect ratio value for a particular sieve cut, a frequency-weighted average aspect ratio was calculated for each of the sieve cuts by summing the product of average aspect ratio and frequency density for a given size class and then normalising for total particle frequency count, as indicated in eq 3. The calculated results, together with the measured BET surface area, are listed in Table 1.

$$
\text { Average Aspect Ratio }=\frac{\sum\left(A R_{x} \cdot f_{x}\right)}{f}
$$

where $A R_{x}$ is the average aspect ratio at size class $x, f_{x}$ is the frequency density at size class $x$ from the area-weighted size distributions (Q2) and $f$ is the total frequency density for the particular sieve fraction. 
Table 1. Calculated Average Aspect Ratio and BET Surface Areas (Standard Deviation) of Different Sieve Fractions of D-mannitol Determined from Dynamic Image Analysis and Nitrogen Adsorption Experiments Respectively

\begin{tabular}{lcc}
\hline Sample $(\mu \mathrm{m})$ & Aspect Ratio $(-)$ & $\mathrm{S}_{\mathrm{BET}}\left(\mathrm{m}^{2} / \mathrm{g}\right)$ \\
& $($ Standard deviation, $\mathrm{n}=3)$ & $($ Standard error $)$ \\
\hline$<75$ & $0.592( \pm 0.0009)$ & $0.5267( \pm 0.0021)$ \\
$75-180$ & $0.547( \pm 0.0002)$ & $0.3590( \pm 0.0018)$ \\
$180-250$ & $0.493( \pm 0.0092)$ & $0.1503( \pm 0.0014)$ \\
$250-425$ & $0.527( \pm 0.0016)$ & $0.1297( \pm 0.0016)$
\end{tabular}

By analysis of the frequency-weighted aspect ratio (Table 1), the average aspect ratios for $<75$ $\mu \mathrm{m}, 75-180 \mu \mathrm{m}, 250-425 \mu \mathrm{m}$ and $180-250 \mu \mathrm{m}$ sieve fractions are respectively $0.592,0.547$, 0.527 and 0.493 . Although the BET surface area decreases as the sieve fraction size increases, the $180-250 \mu \mathrm{m}$ sieve fraction has the lowest average aspect ratio. From the overall downward trend in the aspect ratio, D-mannitol crystals seem to elongate along the c-axis of the crystal lattice as particle size increases. This increase in elongation is consistent with the crystal habit of D-mannitol after continuous growth, although the crystallisation conditions of the commercial sample seem to have hindered such growth in the $180-250 \mu \mathrm{m}$ sieve fractions to a greater extent. Though the crystal shape of the four sieve fractions is similar in the way that they are all prismatic rods, the difference in their elongation means that the proportion of the different crystal faces are not the 
same. Hence, it should be expected that particles with lower aspect ratio should possess a decreasing proportion of the $\{011\}$ face, but an increasing proportion of the $\{120\},\{110\},\{010\}$ and $\{210\}$ face, if they existed on the native crystals.

Surface Free Energy. In our earlier study, the dispersive $\left(\gamma_{S}^{d}\right)$ and polar $\left(\gamma_{S}^{p}\right)$ surface energy components of crystal face $\{010\},\{120\}$ and $\{011\}$ were determined by contact angle measurements on macroscopic size D-mannitol single crystal as shown in Figure 5. The crystal face $\{011\}$ possesses lower $\gamma_{S}^{d}$ but significantly higher $\gamma_{S}^{p}$ compared to facet $\{120\}$ and $\{010\}$, in agreement with the localized surface chemistry of the specific facet from X-ray photoelectron spectroscopic measurements. ${ }^{23}$ The surface energies were determined using purely dispersive diiodomethane and de-ionised water as probe liquids, and then applying Owens-Wendt approach ${ }^{33}$ for surface energy component analysis.
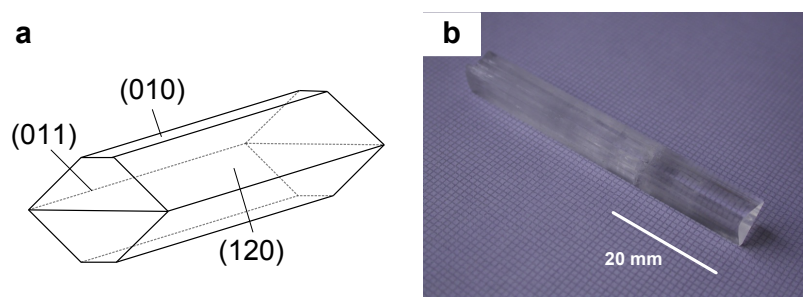

Figure 5. Macroscopic $\beta$ D-mannitol crystal grown from aqueous solution (a) crystal habit and (b) elevated view. 


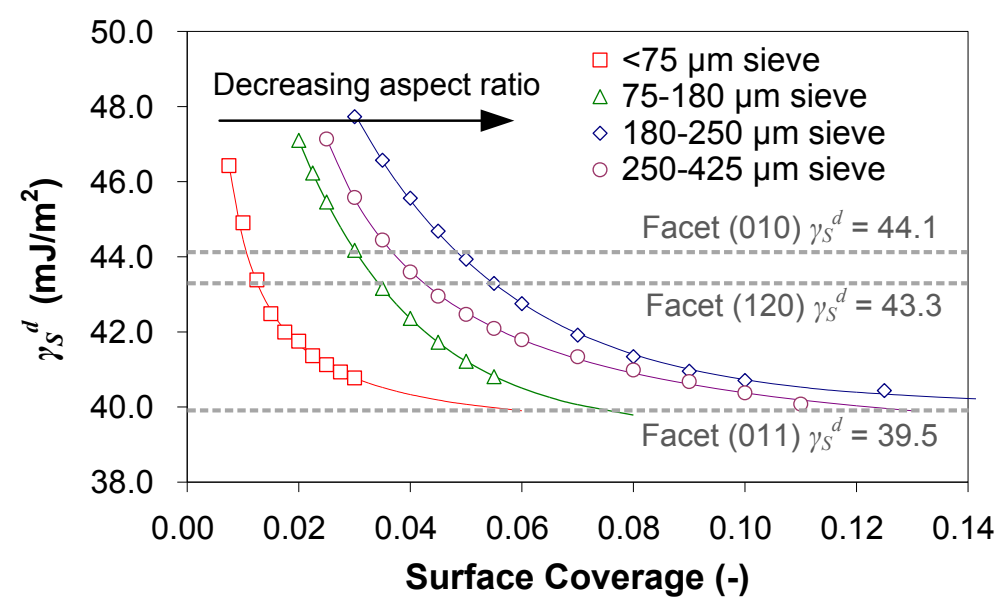

Figure 6. $\gamma_{S}^{d}$ distributions of D-mannitol obtained with different sieve sizes: $<75 \mu \mathrm{m}, 75-180$ $\mu \mathrm{m}, 180-250 \mu \mathrm{m}$ and $250-425 \mu \mathrm{m}$.

The $\gamma_{S}^{d}$ distributions of the four sieve fractions measured using IGC are shown in Figure 6. $\gamma_{S}^{d}$ vary generally between 40 and $48 \mathrm{~mJ} / \mathrm{m}^{2}$ from high to low surface coverage of the probes. It is not surprising that IGC reveals the surface of D-mannitol containing high degree of heterogeneity which can be attributed to the different facets of the crystals, but the dissimilarities in the $\gamma_{S}^{d}$ heterogeneity profiles can be clearly noticed. Although the dispersive surface energies fall within a similar range, the proportion of high and low energy surface sites or regions varies considerably across the four samples. Considering the low energy region (with $\gamma_{S}^{d}<40 \mathrm{~mJ} / \mathrm{m}^{2}$ ), this seems to occupy the highest proportion of the surface on the smallest particles compared to the larger ones. Based on Figure 6, this region occupied at least approximately $94 \%$ of the total surface of the smallest particle fraction. However, this percentage decreased in order of decreasing particle aspect ratio. Referring to Table 1, a decreasing trend in average aspect ratios for the $<75 \mu \mathrm{m}, 75-180 \mu \mathrm{m}$, $250-425 \mu \mathrm{m}$ and $180-250 \mu \mathrm{m}$ sieve fractions was observed. The decrease in the existence of low 
energy sites/regions on the sample as the particle aspect ratio decreases can be explained by the decrease in the proportion of crystal face $\{011\}$ which was found to possess a $\gamma_{S}^{d}$ value close to $39.5 \mathrm{~mJ} / \mathrm{m}^{2}$ from contact angle study. The fact that the $\gamma_{S}^{d}$ distribution is dependent on the aspect ratio, and not on particle size nor the BET surface area, further enhances the hypothesis that the relative exposure of different crystal facets plays a defining role in overall wettability of the sample. The absence of energy regions lower than $\sim 40 \mathrm{~mJ} / \mathrm{m}^{2}$ in Figure 6 indicates that face $\{011\}$ or equivalent might be the lowest $\gamma_{S}^{d}$ crystal plane. When the higher energy region is considered (the surface with $\gamma_{S}^{d}>40 \mathrm{~mJ} / \mathrm{m}^{2}$ ), the proportion of this higher energy surface increases in order of decreasing particle aspect ratio. It is also remarkable that the $\gamma_{S}^{d}$ values of face $\{120\}$ and $\{010\}$ determined from contact angle fall within this $\gamma_{S}^{d}$ range as measured by the IGC. Although the powder sample seemed to contain regions with $\gamma_{S}^{d}$ as high as $48 \mathrm{~mJ} / \mathrm{m}^{2}$, it is speculated that this might represent surface energy of crystal faces which have not been able to measure by contact angle. 


\section{Conclusions}

It has been shown that the facet specific surface energetics for macroscopic crystals of the $\beta$ form of D-mannitol are closely related to the surface energetic profiles for the corresponding powdered crystals. The surface energetic heterogeneity can vary substantially for the same material with different crystal habits. As the aspect ratio of D-mannitol decreased, there was a decreasing shift in the overall contribution of the lower dispersive surface energy sites as measured by the IGC. This was attributed to the decrease in the proportion of the lowest energy facet $\{011\}$. D-mannitol crystals with lower aspect ratio were therefore found to possess higher overall $\gamma_{S}^{d}$. The study has demonstrated the potential of using IGC at finite concentrations to provide an improved understanding of the differences and changes in surface energy for crystals exhibiting dissimilar crystal habits.

Acknowledgements. The authors are grateful for the financial and technical support from AstraZeneca and the access of DIA system in AstraZeneca, Macclesfield. R.H. gratefully acknowledges the useful comments from Dr D.R. Williams. 


\section{References}

(1) Docherty, R.; Clydesdale, G.; Roberts, K. J.; Bennema, P., J. Phys. D: Appl. Phys. 1991, 24 (2), 89-99.

(2) Hartman, P.; Bennema, P., J. Cryst. Growth 1980, 49 (1), 145-156.

(3) Stoica, C.; Verwer, P.; Meekes, H.; van Hoof, P.; Kaspersen, F. M.; Vlieg, E., Cryst. Growth Des. 2004, 4 (4), 765-768.

(4) Chen, J. X.; Wang, J. K.; Ulrich, J.; Yin, Q. X.; Xue, L. Z., Cryst. Growth Des. 2008, 8 (5), 1490-1494.

(5) Davey, R. J.; Black, S. N.; Logan, D.; Maginn, S. J.; Fairbrother, J. E.; Grant, D. J. W., J. Chem. Soc.-Faraday Trans. 1992, 88 (23), 3461-3466.

(6) Winn, D.; Doherty, M. F., AIChE J. 2000, 46 (7), 1348-1367.

(7) Rasenack, N.; Muller, B. W., Int. J. Pharm. 2002, 244 (1-2), 45-57.

(8) Chikhalia, V.; Forbes, R. T.; Storey, R. A.; Ticehurst, M., Eur. J. Pharm. Sci. 2006, 27 (1), 19-26.

(9) Danesh, A.; Connell, S. D.; Davies, M. C.; Roberts, C. J.; Tendler, S. J. B.; Williams, P. M.; Wilkins, M. J., Pharm. Res. 2001, 18 (3), 299-303.

(10) Snyder, R. C.; Veesler, S.; Doherty, M. F., Cryst. Growth Des. 2008, 8 (4), 1100-1101.

(11) Zeng, X. M.; Martin, G. P.; Marriott, C.; Pritchard, J., Int. J. Pharm. 2000, 200 (1), 93-106.

(12) Variankaval, N.; Cote, A. S.; Doherty, M. F., AIChE J. 2008, 54 (7), 1682-1688.

(13) Chow, K.; Tong, H. H. Y.; Lum, S.; Chow, A. H. L., J. Pharm. Sci. 2008, 97 (8), 2855-2877.

(14) Lovette, M. A.; Browning, A. R.; Griffin, D. W.; Sizemore, J. P.; Snyder, R. C.; Doherty, M. F., Ind. Eng. Chem. Res. 2008, 47 (24), 9812-9833.

(15) Heng, J. Y. Y.; Thielmann, F.; Williams, D. R., Pharm. Res. 2006, 23 (8), 1918-1927.

(16) Jaroniec, M.; Madey, R., Physical Adsorption on Heterogeneous Solids; Elsevier: Amsterdam, 1988; pp 4-5.

(17) Begat, P.; Morton, D. A. V.; Staniforth, J. N.; Price, R., Pharm. Res. 2004, 21 (9), 1591-1597.

(18) Lippold, B. C.; Ohm, A., Int. J. Pharm. 1986, 28 (1), 67-74.

(19) Thielmann, F.; Naderi, M.; Ansari, M. A.; Stepanek, F., Powder Technol. 2008, 181 (2), 160-168.

(20) Jones, M. D.; Price, R., Pharm. Res. 2006, 23 (8), 1665-1674.

(21) Yoshinari, T.; Forbes, R. T.; York, P.; Kawashima, Y., Int. J. Pharm. 2002, 247 (1-2), 69-77.

(22) Wade, A.; Weller, P. J. Handbook of Pharmaceutical Excipients. 2nd ed.; American Pharmaceutical Association: Washington, 1994; pp 294-298.

(23) Ho, R.; Hinder, S. J.; Watts, J. F.; Dilworth, S. E.; Williams, D. R.; Heng, J. Y. Y., Int. J. Pharm. 2009, (submitted).

(24) Ticehurst, M. D.; York, P.; Rowe, R. C.; Dwivedi, S. K., Int. J. Pharm. 1996, 141 (1-2), 93-99. (25) Newell, H. E.; Buckton, G.; Butler, D. A.; Thielmann, F.; Williams, D. R., Pharm. Res. 2001, 
$18(5), 662-666$.

(26) Domingue, J.; Burnett, D.; Thielmann, F., Am. Lab. 2003, 35 (14), 32-37.

(27) Schultz, J.; Lavielle, L.; Martin, C., J. Adhes. 1987, 23 (1), 45-60.

(28) Thielmann, F.; Burnett, D. J.; Heng, J. Y. Y., Drug Dev. Ind. Pharm. 2007, 33 (11), 1240-1253.

(29) Yla-Maihaniemi, P. P.; Heng, J. Y. Y.; Thielmann, F.; Williams, D. R., Langmuir 2008, 24 (17), 9551-9557.

(30) Xu, R. L.; Di Guida, O. A., Powder Technol. 2003, 132 (2-3), 145-153.

(31) Kaminsky, W.; Glazer, A. M., Z. Kristallogr. 1997, 212 (4), 283-296.

(32) Walter-Levy, L., Comptes Rendus Hebdomadaries Des Seances De L Academie Des Sciences Serie C 1968, 267, 1779-1782.

(33) Owens, D. K.; Wendt, R. C., J. Appl. Polym. Sci. 1969, 13, 1741-1747. 


\section{For Table of Contents Use Only}

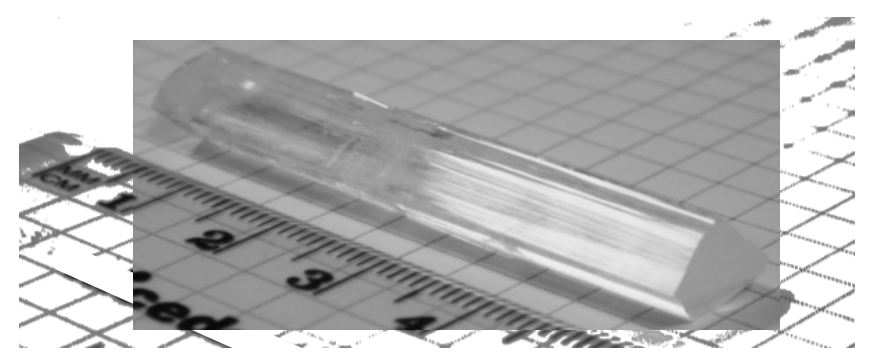

An inverse gas chromatography technique to map the surface energy heterogeneity of D-mannitol powders is employed to derive the relationship between surface energy and varying crystalline habits. 